Aerospace Science and Technology, Volume 91, August 2019, pp. 410-421

DOI:10.1016/j.ast.2019.02.014

\title{
Multi-Objective Optimisation of Short Nacelles for High Bypass Ratio Engines
}

\author{
Fernando Tejero ${ }^{\mathrm{a}, *}$, Matthew Robinson ${ }^{\mathrm{a}}$, David G MacManus ${ }^{\mathrm{a}}$, Christopher \\ Sheaf ${ }^{b}$ \\ ${ }^{a}$ Centre for Propulsion Engineering, School of Aerospace, Transport and Manufacturing, \\ Cranfield University, Bedfordshire, $M K 430 A L$ \\ ${ }^{b}$ Rolls-Royce plc., P.O. box 31, Derby, United Kingdom, DE24 8BJ
}

\begin{abstract}
Future turbo-fan engines are expected to operate at low specific thrust with high bypass ratios to improve propulsive efficiency. Typically, this can result in an increase in fan diameter and nacelle size with the associated drag and weight penalties. Therefore, relative to current designs, there is a need to develop more compact, shorter nacelles to reduce drag and weight. These designs are inherently more challenging and a system is required to explore and define the viable design space. Due to the range of operating conditions, nacelle aerodynamic design poses a significant challenge. This work presents a multi-objective optimisation approach using an evolutionary genetic algorithm for the design of new aero-engine nacelles. The novel framework includes a set of geometry definitions using Class Shape Transformations, automated aerodynamic simulation and analysis, a genetic algorithm, evaluations at various nacelle operating conditions and the inclusion of additional aerodynamic constraints. This framework has been applied to investigate the design space of nacelles for high bypass ratio aero-engines. The multi-objective optimisation was successfully demonstrated for the new nacelle design challenge and the overall system was shown to enable the identification of the viable nacelle design space.
\end{abstract}

Keywords: Optimisation; Nacelle; CFD; Genetic Algorithm. 


\section{Nomenclature}

\section{Roman Symbols}

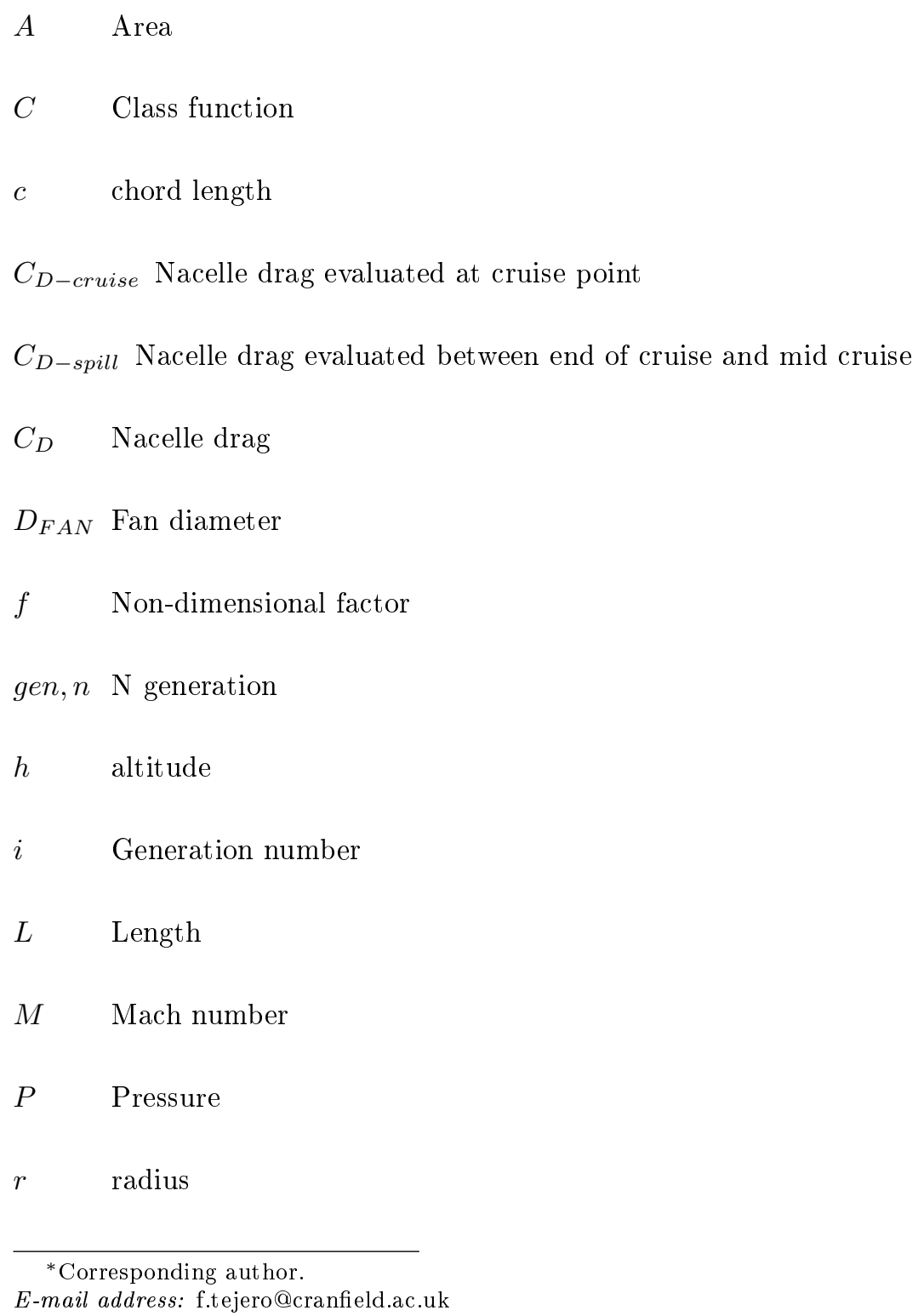




\begin{tabular}{|c|c|}
\hline$S$ & Shape function \\
\hline$T$ & Temperature \\
\hline$V$ & Velocity \\
\hline$x$ & Abscissa \\
\hline \multicolumn{2}{|c|}{ Greek Symbols } \\
\hline$\beta$ & Boat-tail angle \\
\hline$\psi$ & Non-dimensional abscissa \\
\hline$\rho$ & Density \\
\hline$\sigma$ & Mutation size \\
\hline$\xi$ & Non-dimensional ordinate \\
\hline \multicolumn{2}{|c|}{ Superscripts and Subscripts } \\
\hline$\prime \prime$ & Seconde derivative \\
\hline$\infty$ & Freestream \\
\hline$a f$ & Afterbody \\
\hline$D R$ & Drag rise \\
\hline$f b$ & Forebody \\
\hline$h i$ & Highlight \\
\hline if & Initial Forebody \\
\hline $\max$ & Maximum radius \\
\hline$n a c$ & Nacelle \\
\hline
\end{tabular}




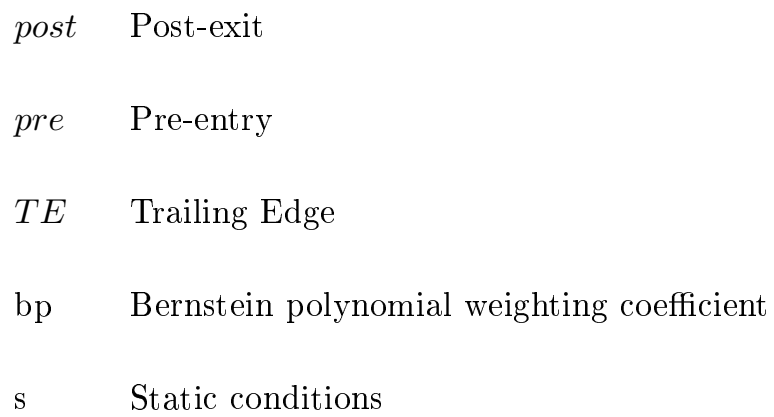

\section{Acronyms}

BPR Bypass Ratio

CDI Circumferential Distortion Index

CFD Computational Fluid Dynamics

CST Class Shape Transformation

FPR Fan Pressure Ratio

GA Genetic Algorithm

iCST Intuitive Class Shape Transformation

MFCR Mass Flow Capture Ratio

MOO Multi-Objective Optimisation

NSGA-II Non-dominated Sorting Genetic Algorithm II

RANS Reynolds-Averaged Navier Stokes

RSM Response Surface Model

SFC Specific Fuel Consumption

SST Shear Stress Transport

VHBR Very High Bypass Ratio 


\section{Introduction}

Reducing operating costs, noise and emissions has been one of the main targets of aero-engine designers over the last decades. In order to reduce the engine specific fuel consumption (SFC) and to improve its propulsive efficiency, there is a general tendency of increasing the bypass ratio (BPR) [1] and reducing the fan pressure ratio (FPR) [2]. It is expected that the future turbofans will employ BPR above 15, which is considerably higher than current configurations $(\mathrm{BPR}=10-11)$. These architectures typically require large engine diameters which can increase the engine weight, nacelle size, and its contribution to the overall aircraft drag [3]. Thus, there is a need to find methods to design viable nacelle geometries without compromising the improvements obtained from the engine cycle [4]. Daggett [5] concluded that the replacement of a turbofan with a $\mathrm{BPR}=7$ and $D_{F A N}=3.175 \mathrm{~m}$ to a geared turbofan with a $\mathrm{BPR}=14.3$ and $D_{F A N}=3.861 \mathrm{~m}$ increased the nacelle drag on a large four engine aircraft by $30 \%$. One of the main challenges in the design of short and slim nacelles is to address the wave drag penalty that arises for typical transonic operations [6]. For example, for a fixed nacelle length $\left(L_{n a c}\right)$, the reduction of the maximum nacelle radius $\left(r_{\max }\right)$ (Fig. 1) results in a greater flow acceleration and ultimately an increase in wave drag. During nacelle design, the highlight radius $\left(r_{h i}\right)$, fan cowl length $\left(L_{n a c}\right)$, maximum nacelle diameter $\left(r_{\max }\right)$ and trailing edge location $\left(r_{t e}\right)$ (Fig. 1) are usually controlled to ensure an acceptable size and shape of the nacelle. While the highlight radius sets the mass flow capture ratio at which the engine operates, the other parameters $\left(L_{n a c}, r_{\max }\right.$ and $\left.r_{t e}\right)$ ensure space for other engine sub-systems which need to be accommodated within the nacelle. The trailing edge location is usually fixed according to engine nozzle requirements as well as to ensure sufficient clearance with the wing and to reduce interference effects between the engine and the airframe. A proper combination of these 
parameters is required to obtain the best achievable aerodynamic performance. Ideally, $L_{n a c}$ and $r_{\max }$ would be reduced as much as possible to minimize the wetted area and fan cowl profile drag. However, this would reduce the fan cowl curvature, which results in a wave drag penalty under transonic conditions. Therefore, a systematic investigation is required to design feasible short nacelles for Very High Bypass Ratio (VHBR) engines.

A significant part of the challenge for nacelle aerodynamic design is the broad range of operating conditions that are encountered throughout the aircraft mission. This includes take-off [7], high incidence [8], crosswind [9], mid-cruise [10], end-of-cruise [11] or windmilling [12]. Nevertheless, for a long-range configuration it is crucial to minimize the nacelle drag during cruise-type conditions. Within the current work, the nacelle design is considered in terms of multipoint conditions around the cruise segment so that the sensitivity to cruise Mach number and massflow capture ratio is addressed. Clearly, one metric of interest, which should be minimized to limit the fuel consumption, is the nacelle drag at mid-cruise conditions $\left(C_{D-\text { cruise }}\right)$. In addition, across the flight profile the engine operating condition changes and therefore the ingested massflow varies accordingly to deliver the required thrust. The mass flow capture ratio (MFCR) is defined as the ratio between the cross-sectional area of the ingested streamtube at an infinite upstream condition and the highlight area. Across the cruise segment of the flight, due to the reduction in MFCR, the stagnation point moves towards the fan engine and the acceleration over the leading edge of the nacelle results in higher peak Mach numbers, a stronger shock wave and a concomitant increase in wave drag [11]. Typically, there is a continuous reduction in the MFCR through the cruise phase and the associated changes in the drag is considered as spillage drag $\left(C_{D-\text { spill }}\right)$ [11]. Another metric of interest during nacelle design is the drag rise Mach number $\left(M_{D R}\right)$. It is typ- 
ically the value at which the nacelle drag rapidly increases as the freestream Mach number $\left(M_{\infty}\right)$ rises. Although an aircraft and engine have a nominal cruise condition and an associated flight Mach number, it is typical to include a design margin in terms of nacelle $M_{D R}$, so an increase of $M_{\infty}$ does not have a large, adverse impact on the nacelle performance. This range of operating conditions and design metrics including $M_{D R}, C_{D-\text { spill }}$ and $C_{D-c r u i s e}$, which are typically considered during nacelle aerodynamic design, highlight the suitability of this problem for multi-objective optimisation (MOO). In addition, a robust and sufficiently flexible parametric representation of the fan cowl geometry is required to carry out the associated shape manipulation. The method should be capable of producing realistic shapes with smooth curves, reproduce a wide variety of different designs with a small number of input variables, be intuitive and be homogenous in the design space. The last point is of vital importance for an optimisation method. It is required that small perturbations in one of the design variables do not have a large impact on the performance metrics. A large number of different parameterization for aerodynamic shapes has been proposed in recent years $[13,14,15]$ and a comprehensive review was presented recently by Masters et al. [16], where it was concluded that the Class Shape Transformation (CST) parameterization method gives an efficient coverage of the design space that was investigated for an aerofoil configuration.

Genetic algorithms (GA) are known to obtain an effective convergence to the Pareto front in complex multi-objective optimisation problems [17]. Albert et al. [18] investigated a multi-objective optimisation (MOO) of intakes and nacelle shapes using a GA. The investigation was focused on different 2D parametric representations to minimize the peak Mach number at cruise $\left(M_{\infty}=0.73\right)$ and static $\left(M_{\infty}=0.05\right)$ conditions simultaneously. It was found that a significant reduction of the peak Mach number could be achieved with an appropriate geo- 
metric parameterization. The comparison of the three different parametric models, super-ellipses and polynomials, CSTs and B-spline, concluded that the CST model was the best suited for the fan cowl description. The work showed that the peak Mach number could be decreased by $14 \%$ at cruise conditions relative to a defined baseline configuration for the CST parametrization. The shape optimisation of an isolated engine nacelle with a GA and two gradient-based methods at cruise $\left(M_{\infty}=0.82\right)$ and crosswind conditions was carried out by Toubin et al. [19]. The investigation was based on a relatively benign nacelle configuration with a long $L_{n a c} / r_{h i}=6.49$. The objective functions for this investigation were the pressure drag coefficient at cruise, neglecting the viscous terms, and the intake circumferential distortion index (CDI) for the crosswind design point. Although the investigation was carried out with Reynolds-Averaged Navier Stokes (RANS) computations, the viscous effects were neglected, motivated by the fact that the nacelle wetted surface was only marginally modified. The performance improvement, in terms of pressure $C_{D}$ and intake circumferential distortion index (CDI), was achieved through a CFD based optimisation. The best trade-off between both objective functions was found for an individual that reduced the pressure $C_{D}$ and CDI by $0.27 \%$ and $3.62 \%$ relative to a baseline nacelle. It was also concluded that additional geometrical constraints should be added in future optimisations to achieve realistic geometries.

Previous studies also investigated nacelle design using surrogate models to reduce the computational expense [10, 20, 21]. Robinson et al. [10] developed a Response Surface Model (RSM) using a Gaussian Processes Regression (Kriging interpolation) [22] as a driver for the GA instead of using CFD simulations. The method was demonstrated to be suitable to assess the design space for nacelle performance at a preliminary stage. The influence of the initial sample, as well as the number of individuals for the subsequent generations, was investigated. 
The potential benefit from using a larger initial generation and reduced overall generations was demonstrated. Fang et al. [20] investigated the aerodynamic optimisation of a transonic nacelle $\left(M_{\infty}=0.80\right)$ based on a hybrid genetic algorithm, combining a Kriging response surface model and numerical simulations (RANS). During the generations, $5 \%$ of the individuals were evaluated with the Kriging response method, which was updated with the increasing of design samples. The single objective optimisation $\left(C_{D-\text { cruise }}\right)$ of short axisymmetric nacelles $\left(L_{n a c} / r_{h i}=3.32\right)$ showed that a reduction of 1.5 aircraft drag counts was achievable. However, the work also emphasized the need to account for other conditions of the flight envelope in future multi-objective optimisations. Lastly, Li et al. [21] optimized the fan cowl shape to delay boundary layer transition under transonic conditions $\left(M_{\infty}=0.78\right)$. A genetic algorithm was used with a Kriging model fitted by 100 CFD samples and the optimal nacelle design predicted by the GA was evaluated by a CFD simulation. Following this approach, it was found a nacelle design in which the area of laminar flow increased by $7 \%$ with respect to a baseline case.

\subsection{Scope of the present work}

Although previous work has addressed different transonic aerodynamic problems such as aerofoils [23], exhaust designs [24] or rotor blades [25], a design approach to provide aero-lines within the new nacelle design space for future aero-engines is not available in the open literature. The overall aim of this work is to present a viable method for the design and multi-objective optimisation (MOO) of compact nacelles. The framework includes a set of geometry definitions using intuitive Class Shape Transformations (iCST), automated aerodynamic simulation and analysis based on CFD, a genetic algorithm, evaluations at various nacelle operating conditions and the inclusion of additional aerodynamic constraints. Nacelles are typically designed as a set of nominally 
axisymmetric aero-lines that are combined, including droop and scarf [26], to produce a full 3D nacelle geometry. However, the key step during this process is the design of each aero-line.

The novelty of this work lies in the development of a multi-objective optimisation design approach, the quantification of the design space characteristics and the identification of the dominant design variables that affect the aerodynamic performance for compact nacelles.

\section{Methods and scope}

\subsection{Methodology Overview}

A fully automatic and integrated framework has been developed for the identification of the viable nacelle design space at given operating conditions. A geometric design tool parametrizes the intake, fan cowl and exhaust-nozzle geometry based on Kulfan's CST functions [27]. The geometry definition is followed by the generation of the multi-block structured computational domain of the engine with an integrated automatic mesh generation tool [28]. The tool automatically sets the user-defined operating conditions, computes the viscous compressible flow-field [29], calculates the performance metrics of interest and repeats the process in a closed-loop following the Genetic Algorithm [30] procedure.

\subsection{Nacelle definition and mesh generation}

Class-Shape-Transformation (CST) curves [27] are usually employed to describe aerodynamic shapes in a parametric manner. They have been mainly applied to airfoil design due to their simplicity compared to other curve definitions [31] but also to complex 3D geometries [32, 33]. CSTs are defined as the

product of a class function, $C(\psi)$, and a shape function, $S(\psi)$, plus a vertical offset between the end points (Eq. 1): 


$$
\xi(\psi)=S(\psi) C(\psi)+\psi \Delta \xi_{T E} ; \xi=\frac{y}{c}, \psi=\frac{x}{c}
$$

where $\xi$ and $\psi$ are the vertical and horizontal coordinates normalized by the chord profile c, i.e. $\xi=\frac{y}{c}$ and $\psi=\frac{x}{c}$. The class function employed during this investigation is the one proposed by Kulfan and Bussoletti [27] for airfoil shapes (Eq. 2). The first term $(\sqrt{\psi})$ ensures an infinite derivative at the leading edge, while the second term (1- $\psi)$ provides a sharp trailing edge.

$$
C(\psi)=\sqrt{\psi}(1-\psi) ; \text { for } 0 \leq \psi \leq 1
$$

A weighted sum of Bernstein polynomials is usually employed as a weighting shape function [34] (Eq. 3). The shape function, which is formed by $n+1$ Bernstein polynomials, is equal to one for unweighted Bernstein coefficients $\left(b_{c i}\right.$ $=1$ ) and can be adjusted to any value when the coefficients are changing.

$$
S(\psi)=\sum_{i=0}^{n} b c_{i}\left(\begin{array}{c}
n \\
i
\end{array}\right) \psi^{i}(1-\psi)^{n-1}
$$

The $n+1$ Bernstein polynomials define a linear set of equations that can be calculated using intuitive design variables as described by Christie et al. [34]. Within this work, a key feature of the proposed shape function definition is that its value at the leading edge is only a function of the local radius of curvature, $r_{i f}$ (Eq. 4), and is related to the boat-tail angle and endpoint offset at the trailing edge (Eq. 5):

$$
\begin{gathered}
S(0)=b c_{0}=\sqrt{\frac{2 r_{i f}}{c}} \\
S(1)=b c_{n}=\tan \left(\beta_{n a c}\right)+\Delta \xi_{T E}
\end{gathered}
$$


Eight design variables were identified to describe the fan cowl: $r_{t e}, r_{h i}$, $L_{n a c}, r_{i f}, r_{\max }, f_{\max }, \beta_{\text {nac }}$, and $y_{T E}^{\prime \prime}$ (Fig. 1). The endpoints of the nacelle are defined by the $r_{t e}, r_{h i}$ and $L_{n a c}$ while the remaining variables $\left(r_{i f}, r_{\max }\right.$, $f_{\text {max }}, \beta_{\text {nac }}$, and $\left.y_{T E}^{\prime \prime}\right)$ set the constraints for the fan cowl CST curve. The initial forebody radius, $r_{i f}$, describes the curvature at the leading edge, the crest location is defined by $r_{\max }$ and $L_{n a c} \cdot f_{\max }$, the boat-tail angle, $\beta_{n a c}$, sets the tangent at the trailing edge, $f^{\prime}\left(x_{T E}\right)=\tan \left(\beta_{n a c}\right)$, and the second derivative at the trailing edge, $f^{\prime \prime}\left(x_{T E}\right)=y_{T E}^{\prime \prime}$, provides an extra control of the afterbody shape to avoid non-monotonic curvature distributions of the fan cowl. For the present investigation, the fan cowl geometry was split in two segments: a 3rd order CST curve for the forebody and a 6th order CST curve for the afterbody. The forebody CST curve was defined using the endpoints (highlight and trailing edge location), the location of the crest ( $r_{\max }$ position) and zero gradient at the crest, $f_{f b}^{\prime}\left(x=f_{\max } \cdot L_{n a c}\right)=0$. The afterbody CST curve employed the previous constraints, ensured 2nd and 3rd derivative continuity with the forebody curve, $f_{a b}^{\prime \prime}\left(x=f_{\text {max }} \cdot L_{n a c}\right)=f_{f b}^{\prime \prime}\left(x=f_{\text {max }} \cdot L_{n a c}\right)$ and $f_{a b}^{\prime \prime \prime}\left(x=f_{\text {max }} \cdot L_{n a c}\right)=f_{f b}^{\prime \prime \prime}\left(x=f_{\text {max }} \cdot L_{n a c}\right)$, and set the second derivative at the nacelle trailing edge, $f_{a b}^{\prime \prime}\left(x=x_{T E}\right)=y_{T E}^{\prime \prime}$. The intake and exhaust system were also defined employing CST curves. The bypass and core ducts were set with the Geometric Engine Modeler Including Nozzle Installation (GEMINI) tool $[35,24]$ which is able to create an axisymmetric separate-jet exhaust system for any designated engine cycle. Within this work, a generic exhaust has been used to provide a representative exit streamtube so that the nacelle post exit terms are appropriate. In this context, the focus of this investigation is on the nacelle drag to ensure consistency with the thrust-drag accounting methods employed (Section 2.3.3).

The bounds of each nacelle design variable were initially set to avoid non- 
monotonic curvature distributions on the fan cowl geometries. After each optimisation routine, the non-dominated individuals were represented with a parallel coordinates plot to ensure that the design variables bounds had been selected properly (Section 3). Table 1 summarizes the lower and upper bounds for each design variable employed in the parametrization of the fan cowl, where $f_{i f}$ corresponds to the non-dimensional value of the the initial forebody radius $\left(r_{i f}\right)$ and is defined as $f_{i f}=r_{i f} \frac{f_{\max } L_{n a c}}{\left(r_{\max }-r_{h i}\right)^{2}}$.

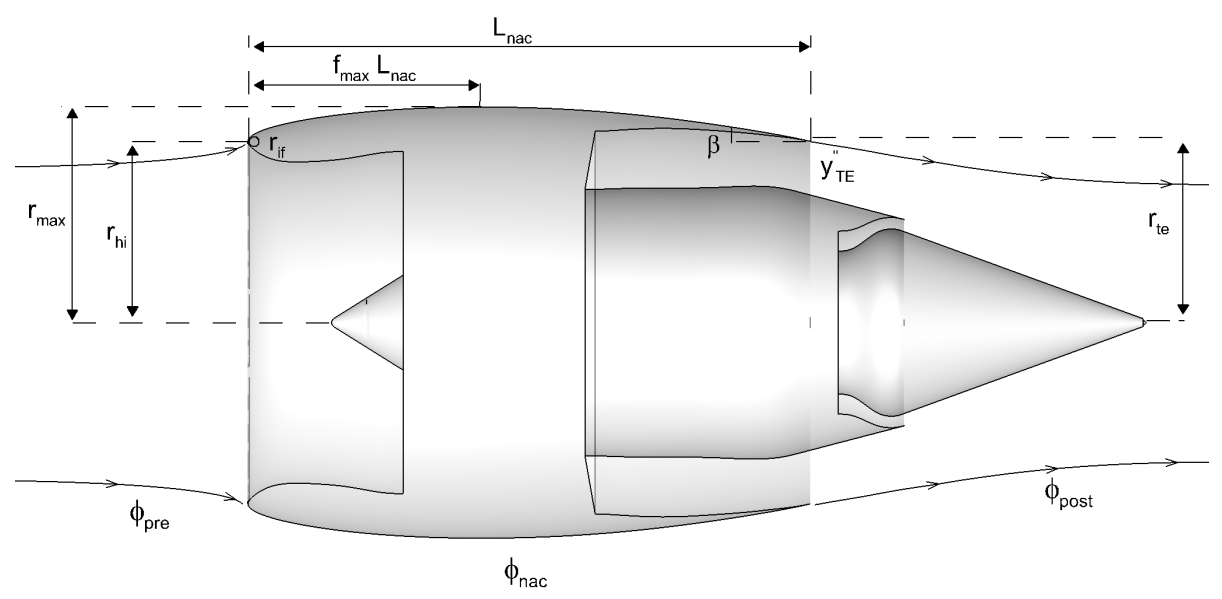

Figure 1: Nacelle geometry parametrisation of the nacelle and thrust and pre-enty and postexit streamtubes 


\begin{tabular}{ccc}
\hline Variable & Lower Bound & Upper Bound \\
\hline$f_{\max }[-]$ & 0.3 & 0.5 \\
$r_{\max } / r_{h i}[-]$ & 1.1 & 1.3 \\
$f_{i f}[-]$ & 0.6 & 1.5 \\
$y_{T E}^{\prime \prime} / r_{h i}[-]$ & -0.5 & 0.0 \\
$\beta_{\text {nac }}\left[{ }^{\circ}\right]$ & 9.0 & 15.0 \\
\hline
\end{tabular}

Table 1: Bounds of the fan cowl design variables during the optimisation routine

\subsection{Computational method}

\subsubsection{Flow solver and boundary conditions}

The compressible steady Favre-averaged Navier-Stokes equations were solved using a double precision implicit density-based solver, a second order spatial discretization with Green-Gauss node based scheme and the $k-\omega$ SST turbulence model closure [29]. For all simulations, the convergence criteria was based on a reduction of normalised residuals of five order of magnitude and an oscillation of the total force on the fan cowl lower than $0.05 \%$ over the last 500 iterations. The freestream conditions were specified by setting the static pressure, $P_{s}$, static temperature, $T_{s}$ and Mach number $M$. The intake massflow was controlled with a pressure-outlet condition, which fixed the mass flow capture ratio (MFCR) of the engine. The fan and low-pressure turbine exit were defined as pressure-inlets by total pressure and temperature, $P_{t}$ and $T_{t}$. As the present investigation is focused on the drag domain, the aerodynamic conditions at the fan and low pressure turbine exit were set using an ideal isentropic expansion to minimise exhaust dependent interference on the nacelle drag.

To reflect the expected operating conditions for the future turbofan engines, the cruise conditions were set to a freestream Mach number of $M_{\infty}=0.85$ with a MFCR $=0.70$ at $h=10668 \mathrm{~m}$. During this investigation, the spillage drag was defined as the increase of drag between start and end of cruise with an MFCR $=0.70$ and 0.63 , respectively.

An automated structured grid generation process was implemented in the 
framework and domain and grid independence studies were carried out. Four different domain sizes $\left(40 r_{\max }, 60 r_{\max }, 80 r_{\max }\right.$ and $\left.100 r_{\max }\right)$ were considered and across this range the cruise nacelle drag increased monotonically with a $0.10 \%$ reduction between the $80 r_{\max }$ and $100 r_{\max }$ configurations. Finally, the $80 r_{\max }$ domain size was employed throughout this study. The grid convergence was calculated for four different mesh sizes (10k, 20k, 40k and 80k), with boundary layer blocks discretized to satisfy a $y^{+}$of approximately 50 so that the boundary layers were resolved by the wall function model implemented in the $k-\omega$ SST turbulence closure. A key aspect within an optimisation framework based on a GA with CFD in-the-loop is to enable a tractable investigation with sufficient populations in each generation as well as convergence across the required number of generations. The solutions using the 20k mesh demonstrated a grid convergence index [36] of $1 \%$ on $C_{D}$ at cruise conditions $\left(M_{\infty}=0.85\right.$ and MFCR $=0.70)$ and, considering the GA requirements, was adopted within this study.

\subsection{2. $C F D$ validation}

The performance of the CFD approach was tested on an axisymmetric nacelle geometry to determine the accuracy of the computational model. The open source ARA cowl 1, which was designed to assess alternatives to the conventional NACA series 1 type forebody designs, was selected for the validation [37]. It has a cylindrical centrebody with a circular arc and straight line for the afterbody. The measurements covered a wide range of different aerodynamic points varying the freestream Mach number from 0.65 to 0.95 and MFCR from 0.40 to 0.76 . In total 62 aerodynamic conditions were simulated and compared with the available experimental data. The CFD investigation was carried out for different mesh sizes $(10 \mathrm{k}, 20 \mathrm{k}, 40 \mathrm{k}$ and $80 \mathrm{k})$ and $y^{+}$of approximately 50 using wall functions for the boundary layer treatment to be consistent with the 
method used in the optimisation process. For the selected mesh employed during the optimisation studies (20k), the cruise drag was typically within $3.5 \%$ of the measured data cruise $C_{D}$ at both MFCR $=0.70$ and 0.60 (Fig. 2a and 2b). In addition, the CFD accuracy for drag rise divergence $M_{D R}$ (Eq. 6) was $\Delta M_{D R}=-0.003$ at MFCR $=0.7$, with an experimental $M_{D R}=0.848$. The discrepancy increased to $\Delta M_{D R}=0.005$ at $\mathrm{MFCR}=0.60$, with a measured $M_{D R}=0.826$. The $M_{D R}$ for experimental and CFD data shows that as the MFCR decreases, the drag divergence occurs at a lower Mach number. This effect is caused by the movement of the stagnation point and resultant acceleration of the flow increasing at the highlight for a lower MFCR. A lower MFCR causes the stagnation point on the nacelle to move closer to the throat and therefore the flow has a greater distance to travel around the lip. Since this region is highly curved a strong acceleration occurs which gives a higher peak Mach number and results in a stronger shock wave. This results in an earlier onset of drag divergence due to increased local forebody overspeed. The CFD simulations slightly under-predicted the influence of the spillage drag measured at lower MFCRs. For example, the experimental nacelle drag coefficient $C_{D}$ increased from 0.036 to 0.064 when the MFCR was reduced from 0.78 to 0.51 at $M_{\infty}=0.85$ to give $C_{D-\text { spill }}=0.028$. Across the same range of MFCR, the CFD predicted a $C_{D-\text { spill }}$ of 0.026 for the selected grid size of 20k (Fig. 2c). Overall the CFD approach, with the selected mesh resolution and domain size, provides sufficient agreement with the measurements to provide confidence that it can be used in the optimisation studies.

$$
\frac{\partial C_{D}}{\partial M_{\infty}}=0.1
$$




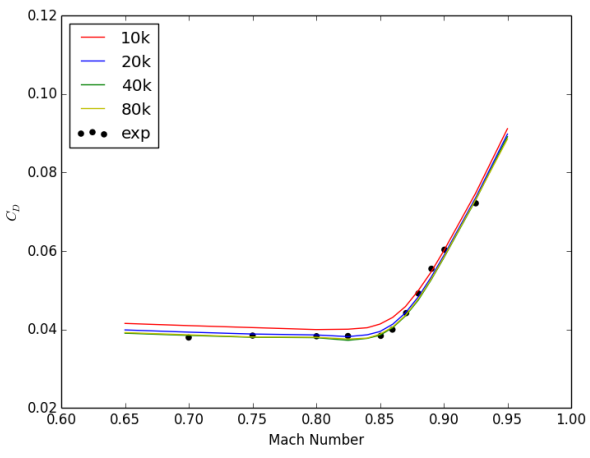

(a) $\mathrm{MFCR}=0.70$

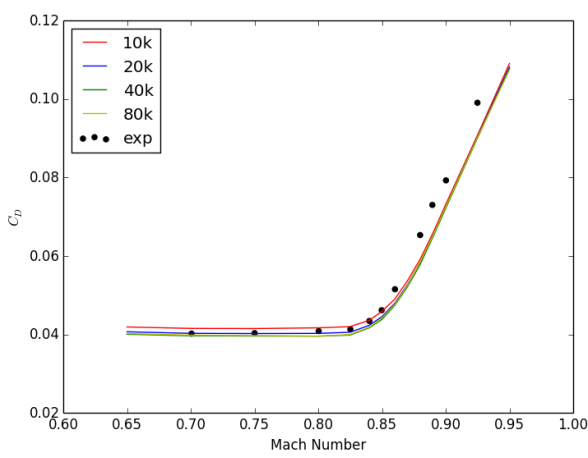

(b) $\mathrm{MFCR}=0.60$

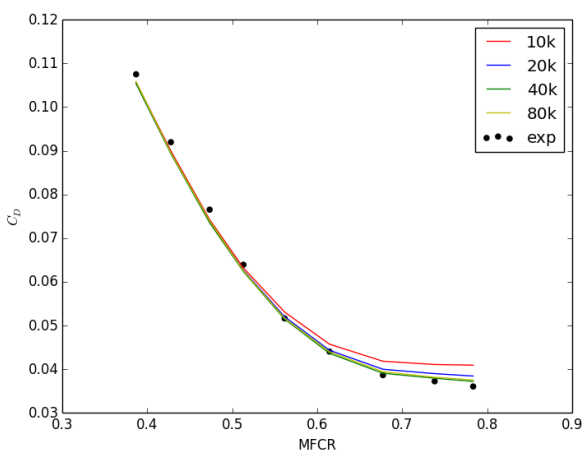

(c) $M_{\infty}=0.85$

Figure 2: ARA cowl 1 validation: Mach sweep at (a) MFCR $=0.7$ and (b) $\mathrm{MFCR}=0.60$ and MFCR sweep at (c) $M_{\infty}=0.85$ 


\subsubsection{Drag extraction}

A consistent thrust drag bookkeeping (TDB) method for thrust and drag decomposition is required to evaluate the performance of an aircraft. Within this work, the nacelle aerodynamic drag (Eq. 7) is calculated using the thrustdrag bookkeeping from AGARD [38]:

$$
D_{n a c}=\phi_{\text {nac }}+\phi_{\text {pre }}+\phi_{\text {post }}
$$

where $\phi_{n a c}$ refers to the pressure and viscous forces that act on the fancowl, $\phi_{\text {pre }}$ is the pre-entry force and $\phi_{\text {post }}$ refers to the post-exit force (Fig. 1).

The post-exit force $\left(\phi_{\text {post }}\right)$ is calculated by pressure integration of the streamline from the engine trailing edge that divides the drag and thrust domains, $\phi_{n a c}$ is extracted by the integration of the forces, pressure and viscous, along the nacelle and the pre-entry force $\left(\phi_{\text {pre }}\right)$ is calculated with the modified nearfield method as described by Christie et al. [39]. This method relies on the momentum integration of the stream tube at upstream infinity and at the fan face and includes the force integration, pressure and viscous term, at the intake and spinner.

\subsubsection{Optimisation algorithm}

In the majority of engineering problems, several objective functions have to be considered. Therefore, it is required to find efficient approaches to converge the solution to a Pareto front. Nacelle design is a clear example of multiobjective optimisation with different performance metrics $\left(C_{D-\text { cruise }}, M_{D R}\right.$ and $\left.C_{D-\text { spill }}\right)$ at different flow conditions. The non-dominated sorting genetic algorithm (NSGA-II) [30] has been implemented in the described framework for the current study. The population is sorted by dominance into non-dominated sets. Diversity is ensured through the use of a distance operator which penalizes clus- 
tered individuals. The proposed approach evaluates the objective functions and constraints of each individual by means of CFD simulations. The optimisation was carried out with a Gaussian mutation operation with a mutation rate of 0.2 and mutation size of $\sigma=0.0005$. The crossover used the $B L X-\alpha$ operator with $\alpha=0.5$ which results in an even split between the two parent designs. A statistical approach with a sample size of 50 to determine the influence of the initial seed in the Pareto optimal set was investigated previously [10]. A zeroth generation of 400 individuals, $i_{g e n, 0}=400$, improved the median hypervolume [10] compared to $i_{g e n, 0}=40$, reducing considerably the scatter in the results. For the purpose of this work, the Latin Hypercube Sampling (LHS) zeroth generation was set to 400 individuals, $i_{g e n, 0}=400$, with 50 individuals in the subsequent generations, $i_{g e n, n}=50$, over a total of 30 generations to ensure the convergence to the Pareto front. Therefore, a total of 1850 different nacelle geometries were evaluated for each specified configuration of $L_{n a c} / r_{h i}$ and $r_{t e} / r_{h i}$. To measure the convergence and diversity of the Pareto front solution, a hypervolume indicator was calculated through the generations. The increase of the hypervolume was lower than $1 \%$ for the last 3 generations which ensured convergence to the Pareto front.

Although previous work has indicated that the exhaust performance can be sensitive to the nacelle trailing edge $\beta_{\text {nac }}[35]$, the aim in this work is to evaluate the nacelle drag within the context of the thrust-drag bookkeeping accounting process. The impact of the nacelle design on the exhaust has not been evaluated as the initial need is for more generic nacelle drag data independent of the exhaust system design. For the present optimisation routine, three nacelle performance metrics and one aerodynamic metric were employed as objective functions. The nacelle cruise drag $\left(C_{D-\text { cruise }}\right)$ (Eq. 8) was calculated following the drag extraction method described in Section 2.3.3 [40]. Although the 
drag rise Mach number $\left(M_{D R}\right)$ is a key factor for nacelle design, this metric was replaced by a change in nacelle drag $\left(\Delta C_{D-M a c h}\right)($ Eq. 9$)$ at cruise Mach number $\left(M_{\infty}\right)$ and an increased conditions of $M_{\text {delta }}=M_{\infty}+0.02$. The third performance metric was the spillage drag $\left(C_{D-\text { spill }}\right)$ (Eq. 10$)$ defined as the change of nacelle drag between mid-cruise and end-of-cruise conditions. Lastly, the number of points at which the gradient changed polarity in the pressure distribution was quantified and this aerodynamic metric minimized. In addition, a constraint on the peak isentropic Mach number along the fan cowl was set to $M_{i s}<1.30$ to avoid undesirable nacelle designs with excessive acceleration and potentially adverse boundary layer interactions.

$$
\begin{gathered}
C_{D-\text { cruise }}=\frac{D_{n a c}}{\frac{1}{2} \rho_{\infty} V_{\infty}^{2} A_{h i}} \\
\Delta C_{D-M a c h}=\frac{D_{\text {nac }, M=M_{\infty}+0.02}-D_{n a c, M=M_{\infty}}}{\frac{1}{2} \rho_{\infty} V_{\infty}^{2} A_{h i}} \\
C_{D-\text { spill }}=\frac{D_{\text {nac }, M F C R_{\text {cruise }}-D_{n a c, M F C R_{E O C}}}}{\frac{1}{2} \rho_{\infty} V_{\infty}^{2} A_{h i}}
\end{gathered}
$$

\section{Results and analysis}

\subsection{Preliminary multi-objective optimisation}

Before performing a thorough investigation of the viable design space of short nacelles for future aero-engines, a reference case with a relatively challenging configuration of $L_{n a c} / r_{h i}=2.4$ and $r_{t e} / r_{h i}=1.0$ was considered. The main aim was to evaluate the capabilities of the developed framework for nacelle multiobjective optimisation. Nacelle aerodynamic design is a complex multi-objective optimisation with an inherently nonlinear nature [10]. This is highlighted in Fig. $3 \mathrm{a}$, in which the result of the optimisation process over 30 generations provides 
a three-dimensional Pareto surface. Figure 3a illustrates the projection of this Pareto in the $C_{D-\text { cruise }}-\Delta C_{D-M a c h}$ space, and colored by $C_{D-\text { spill }}$, where the dominated and non-dominated individuals are marked by "+" and "o", respectively. Throughout the optimisation process there was a $10.3 \%$ range in the nacelle cruise drag $\left(C_{D \text {-cruise }}\right)$ between the individuals with lower and higher cruise drag of the Pareto front, varying from $C_{D \text {-cruise }}=0.0253$ to $C_{D-\text { cruise }}=0.0279$. On the other hand, the spillage changed from $C_{D-\text { spill }} \approx 0$ to $C_{D-\text { spill }}=0.0018$ within the non-dominated individuals. All non-dominated fan cowl designs exhibit a $C_{D-\text { spill }}<0.1 \cdot C_{D-\text { cruise }}$, which is usually acceptable during nacelle design. The individual with the lowest $C_{D \text {-cruise }}$ was reduced by $4.2 \%$ throughout the optimisation routine, changing from $C_{D-\text { cruise }}=0.0264$ at the initial generation to $C_{D-\text { cruise }}=0.0253$ at the end of the optimisation routine.

The performance metrics for the individuals with the lowest $C_{D-c r u i s e}$, $\Delta C_{D-M a c h}$ and $C_{D-\text { spill }}$ (A1, A2, A3, respectively in Fig. 3a) are summarized in the Table 2. Relative to A1 which has the lowest cruise drag $\left(C_{D \text {-cruise }}\right.$ $=0.0253$ ), $\mathrm{A} 2$ has almost a $50 \%$ reduction in $\Delta C_{D-M a c h}$ (from 0.0150 to $0.0077)$; no notable change in $C_{D-\text { spill }}$, but at the expense of a $6.9 \%$ increase in $C_{D \text {-cruise }}$. On the other hand, $\mathrm{A} 3$ can achieve effectively no spillage drag $\left(C_{D-\text { spill }} \approx 0\right)$, at the expense of a $3.5 \%$ increase in cruise drag and also a modest improvement in $\Delta C_{D-M a c h}$ from 0.0150 to 0.0147 . In terms of the Mach number distributions at cruise conditions, all three example designs show the same forward loaded distribution with the same peak Mach number magnitude, $M_{i s}=1.3$, located at about $0.04 X / L_{n a c}$ (Fig. 3b, 3c and 3d). Downstream of the peak suction location there are noticeable differences in the lift distributions for the highlighted designs. Design A1 has a smooth, monotonic reduction in $M_{i s}$ (Fig. $3 \mathrm{~b}$ ), which is characteristically different from designs A2 and A3 


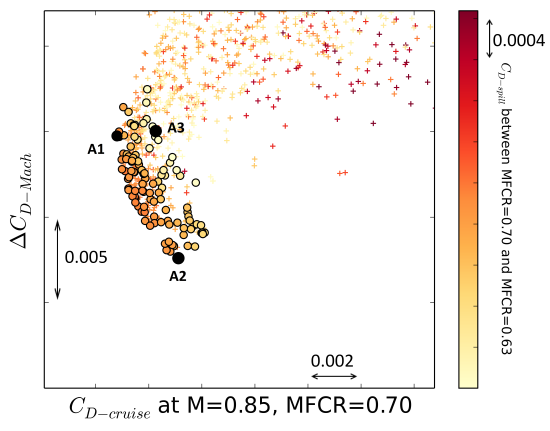

(a) Pareto front

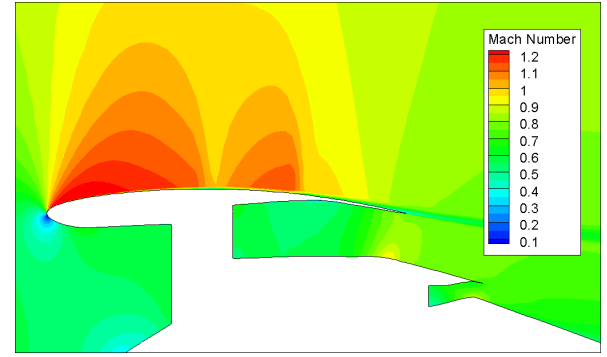

(c) Mach contours for Sample A2

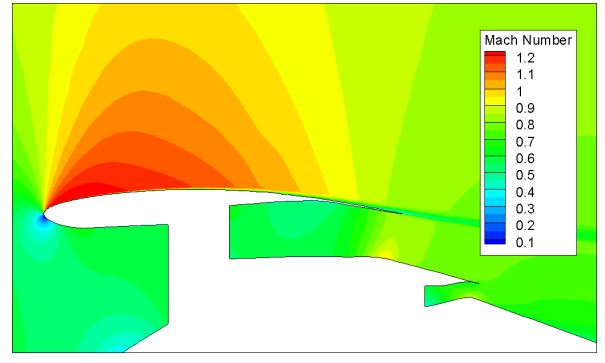

(b) Mach contours for Sample A1

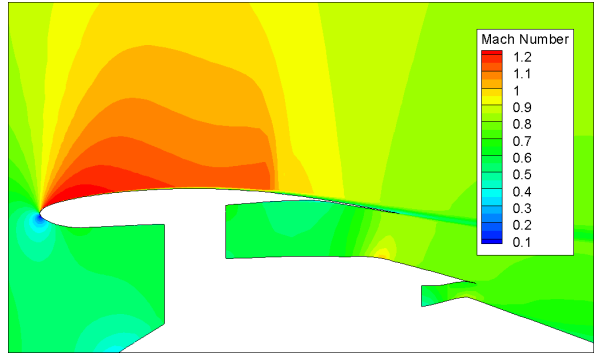

(d) Mach contours for Sample A3

Figure 3: (a) Pareto front surface for $L_{n a c} / r_{h i}=2.4$ and $r_{t e} / r_{h i}=1.0$ and Mach number contour maps for (b) Sample A1, (c) Sample A2 and (d) Sample A3 at $M_{\infty}=0.85$, MFCR $=0.7$ with isentropic expansion at the nozzle to minimise exhaust dependent interference on the nacelle drag

which have well-defined shocks. The example A2 presents a double shock wave topology (Fig. 3c), which has associated large shock losses, making this design the one with the largest cruise drag. A2 has a relatively weak first shock which is located just downstream of the nacelle maximum thickness and with a preshock $M_{i s}$ of 1.12 at $X / L_{n a c}=0.41$. The flow re-accelerates over the aft body downstream of the nacelle crown and results in a second shock with an increased pre-shock $M_{i s}$ of 1.15 . The design A3, which has the lowest spillage $C_{D-\text { spill }}$, presents a $M_{i s}$ distribution with a single normal shock and a pre-shock $M_{i s}$ of about 1.15 at $X / L_{n a c}=0.62$ (Fig. $3 \mathrm{~d}$ ). This configuration results in the least sensitivity to MFCR with a $C_{D-\text { spill }} \approx 0$ (Table 2$)$.

When the freestream Mach number is increased to $M_{\infty}=0.87$ at the same 


\begin{tabular}{lccc}
\hline & $C_{D-\text { cruise }}$ & $\Delta C_{D-\text { Mach }}$ & $C_{D-\text { spill }}$ \\
\hline Individual A1 & 0.0253 & 0.0150 & 0.0015 \\
Individual A2 & 0.0271 & 0.0077 & 0.0015 \\
Individual A3 & 0.0262 & 0.0147 & $\approx 0$ \\
\hline
\end{tabular}

Table 2: Performance metrics for the designs with the lowest $C_{D-\text { cruise }}, \Delta C_{D-M a c h}$ and $C_{D-\text { spill }}$

MFCR $=0.70$, all three designs present similar lift distributions (Fig. 4a, 4b and $4 \mathrm{c}$ ). A strong shock wave is formed at $X / L_{n a c}=0.62$ with a pre-shock $M_{i s}$ of 1.22. 1.19 and 1.22 for the designs A1, A2 and A3, respectively. For the design A1, the change of MFCR from 0.70 to 0.63 at a flight Mach number of 0.85 reveals a first shock wave at $0.41 X / L_{n a c}$ with a pre-shock $M_{i s}$ of 1.15 and a second weak shock at $0.61 X / L_{n a c}$ with a pre-shock $M_{i s}$ of 1.10 (Fig. 4a). Similar double shock structure is found for the design A2, but with a stronger pre-shock $M_{i s}$ of 1.15 for the second shock wave (Fig. 4b). The design A3 gives a smooth, monotonic reduction in $M_{i s}$, in contrast to the single normal shock presented at cruise $\left(M_{\infty}=0.85\right.$ and MFCR $\left.=0.70\right)$, which results in the best design in terms of $C_{D-\text { spill }}$ (Fig. 4c). The nacelle drag across a range of freestream Mach numbers from 0.81 to 0.89 at constant MFCR $=0.70$ was assessed for each sample. The calculated $M_{D R}$ (Eq. 6) was $0.853,0.863$ and 0.852 for the designs A1, A2 and A3, respectively. The sample A2, which has the lowest $\Delta C_{D-M a c h}$, presented the largest $M_{D R}$, while the other two designs A1 and A3 have similar values of $\Delta C_{D-M a c h}$ as well as $M_{D R}$ (Fig. $4 \mathrm{~d}$ ).

It has been demonstrated that the proposed tool evaluates the nacelle aerodynamics within the context of cruise drag as well as off-design multi-point conditions such as low MFCR and different flight Mach numbers. The capabilities of the proposed framework to identify a set of optimum nacelle designs in terms of the performance metrics of interest, i.e. $C_{D-\text { cruise }}, \Delta C_{D-M a c h}$ and $C_{D-\text { spill }}$, has been shown. Hence, the framework can be employed with confidence to 


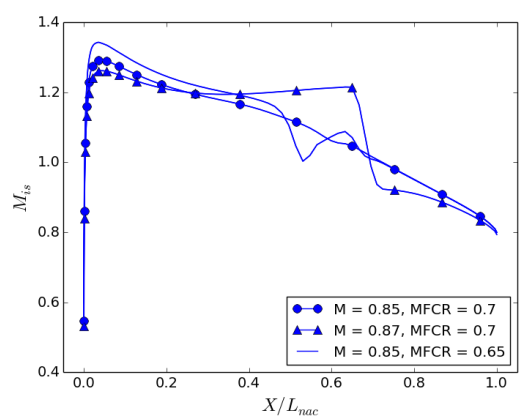

(a) Sample A1

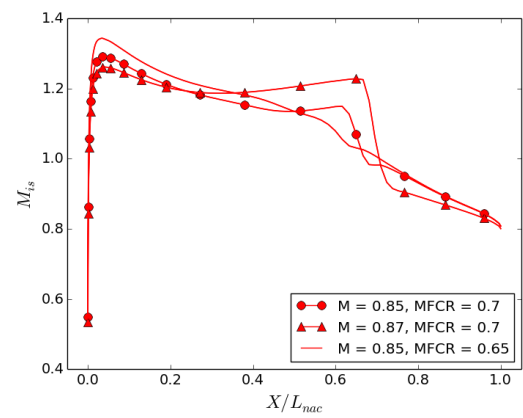

(c) Sample A3

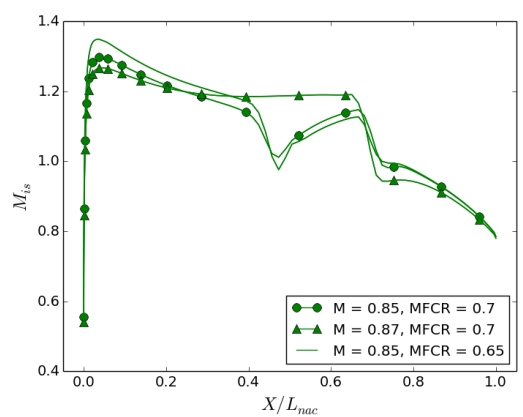

(b) Sample A2

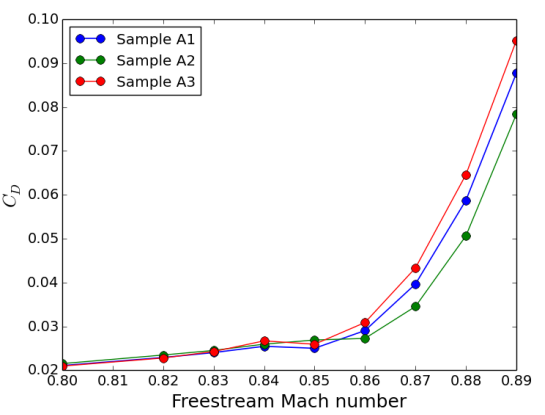

(d) Mach sweep at MFCR $=0.70$

Figure 4: Isentropic distribution at cruise conditions, $\Delta M\left(M_{\infty}+0.02\right)$ and EOC for (a) Sample A1, (b) Sample A2 and (c) Sample A3. Nacelle drag coefficient as a function of the freestream Mach number at (d) MFCR $=0.7$ 
conduct an extensive numerical investigation on the aerodynamic behavior of short nacelles for VHBR engines. The results of a Design Space Exploration (DSE) highlights the feasible parts of the design space for short nacelles and can be used as guidelines in the early stage of the design process.

\subsection{DSE for short nacelles of future turbo-fan engines}

To demonstrate the ability of the method for the particular considerations of nacelle aerodynamic design, and to identify the aerodynamic characteristics for new compact nacelles, the investigation was extended to short nacelle aerolines across the range of $2.4<L_{n a c} / r_{h i}<3.0$ and $0.89<r_{t e} / r_{h i}<1.0$. The nacelle leading edge was fixed, and the trailing edge varied depending on the configuration investigated. Across the overall design space considered, a full factorial assessment of 12 configurations was performed using four $L_{n a c} / r_{h i}(2.4,2.6$, 2.8 and 3.0) and three $r_{t e} / r_{h i}(0.89,0.95$ and 1.0). For each configuration of $L_{n a c} / r_{h i}$ and $r_{t e} / r_{h i}$ a MOO optimisation was carried out with the established method of $i_{g e n, 0}=400$ and 30 subsequent generations of 50 evaluations each.

\subsubsection{Feasibility of the design space}

The MOO of the 12 configurations considered in this work to investigate the trade-off between $L_{n a c} / r_{h i}$ and $r_{t e} / r_{h i}$ for compact aero-engines revealed that the spillage was always within a potentially acceptable range for nacelle design $\left(C_{D-\text { spill }}<0.1 \cdot C_{D-\text { cruise }}\right)$. Therefore, the subsequent analysis is based on the cruise drag and the sensitivity to flight Mach number. In this respect, the obtained set of 3D optimal Pareto fronts, which are similar to the one shown in Fig. 3 , is only presented in the $C_{D-\text { cruise }}-\Delta C_{D-\text { Mach }}$ space which allows for an easier comparison of the projected Pareto fronts (Fig. 5). This comparison highlights the changes in the feasibility of the design space for the pertinent design variables of $L_{n a c} / r_{h i}$ and $r_{t e} / r_{h i}$. For example, for a fixed fan cowl 
length with $L_{n a c} / r_{h i}=3.0$, the minimum $C_{D-\text { cruise }}$ increases by $9.2 \%$ when the trailing edge $r_{t e} / r_{h i}$ moves from 1.0 to 0.89 . This tendency is caused by the expected higher Mach number along the fan cowl as the trailing edge moves inboards towards $r_{t e} / r_{h i}=0.89$ due to the increment of fan cowl curvature. In contrast to the more conventional $L_{n a c} / r_{h i}=3.0$ configuration, when the fan cowl length is shortened to $L_{n a c} / r_{h i}=2.6$ the minimum $C_{D-c r u i s e}$ increases by $35.0 \%$ in the range of $r_{t e} / r_{h i}$ from 1.0 to 0.89 . It highlights the influence of the trailing edge location as nacelles are shortened. For example, a 13.3\% reduction in nacelle length, from $L_{n a c} / r_{h i}=3.0$ to $L_{n a c} / r_{h i}=2.6$, increases the sensitivity of minimum $C_{D-\text { cruise }}$ across $r_{t e} / r_{h i}$ by a factor of four, from $9.2 \%$ to $35.0 \%$. A detailed description of the influence of fan cowl length and trailing edge location as well as guidelines for the design of compact nacelles is reported in the next sections. 


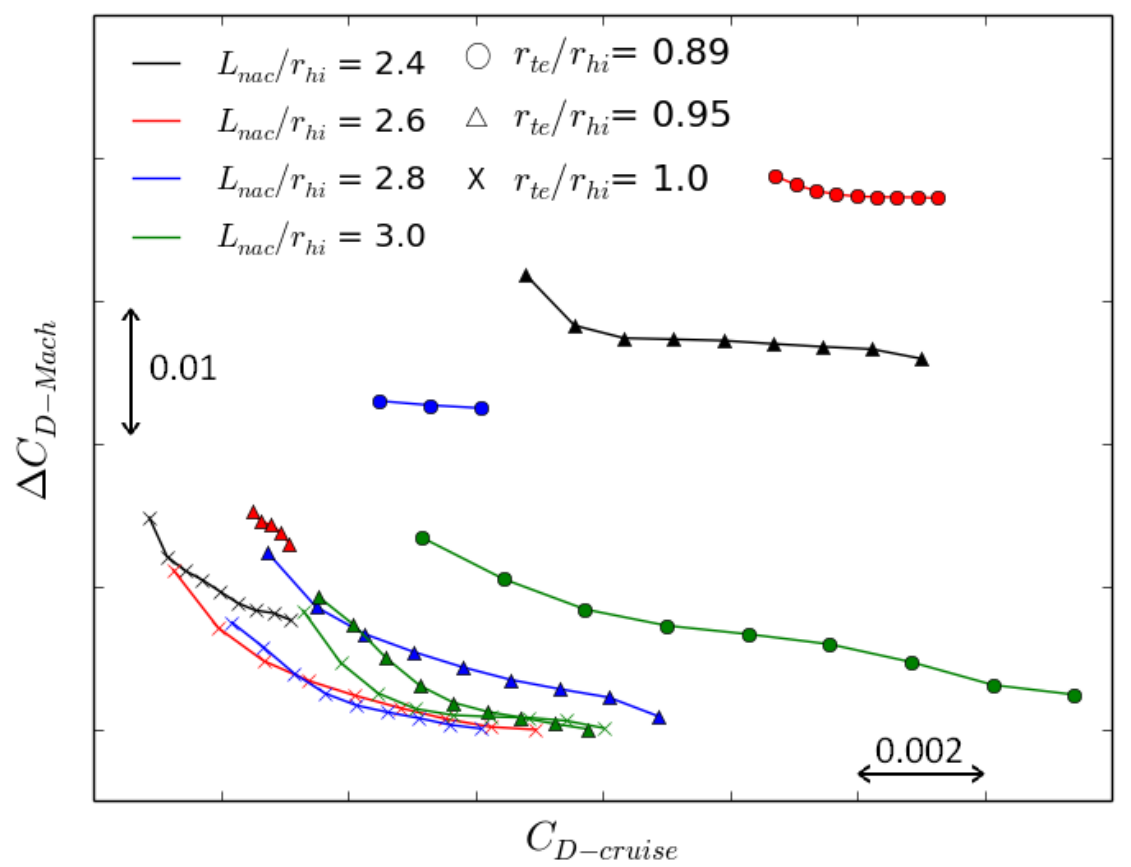

Figure 5: Projection of the Pareto in the $C_{D-\text { cruise }}-\Delta C_{D-M a c h}$ space for VHBR engines

\subsubsection{Influence of $L_{n a c} / r_{h i}$ and $r_{t e} / r_{h i}$}

After establishing a method to investigate the feasible regions of the design space for high bypass ratio aero-engines by means of MOO, the influence of fan cowl length, $L_{n a c} / r_{h i}$, and trailing edge location, $r_{t e} / r_{h i}$, in the performance metrics of $C_{D-\text { cruise }}$ and $\Delta C_{D-M a c h}$ has been quantified and the trade-offs between both parameters identified.

For the relatively conventional fan cowl length of $L_{n a c} / r_{h i}=3.0$, the lowest nacelle drag presents a $C_{D-\text { cruise }}=0.0273,0.0279$ and 0.0293 for $r_{t e} / r_{h i}=$ 1.0, 0.95 and 0.89 , respectively (Fig. 6a). For these designs the $\Delta C_{D-M a c h}$ increases from 0.0075 to 0.0121 as $r_{t e} / r_{h i}$ reduces from 1.0 to 0.89 (Fig. 6b). While some designs with $\Delta C_{D-M a c h} \approx 0$ and $C_{D-\text { cruise }} \approx 0.0319$ were found for 
the trailing edge locations of $r_{t e} / r_{h i}$ of 1.0 and 0.95 , the lowest $\Delta C_{D-M a c h}$ for a more compact nacelle of $r_{t e} / r_{h i}=0.89$ was 0.0051 . Nevertheless, this design had associated a $C_{D-\text { cruise }}=0.0350$, which is a $19.5 \%$ greater with respect to the minimum cruise drag $\left(C_{D-\text { cruise }}=0.0293\right)$ found for this configuration throughout the MOO. This highlights that for a fixed fan cowl length there are more inherent challenges to design aerodynamically favorable designs for compact nacelles. For this $L_{n a c} / r_{h i}$ configuration $\left(L_{n a c} / r_{h i}=3.0\right)$, there is a $9.2 \%$ increase of the minimum cruise drag in the range of $r_{t e} / r_{h i}$ from 1.0 to 0.89 (Fig. 6a).

Another key consideration for the design of compact nacelles is the sensitivity to $L_{n a c} / r_{h i}$. For a fixed $L_{n a c} / r_{h i}=2.8$, the nacelle design with minimum cruise drag increases by $19.3 \%$ in the range of $r_{t e} / r_{h i}$ from 1.0 to 0.89 (Fig. 6a), which is significantly larger than the $9.2 \%$ variation for the more conventional nacelle of $L_{n a c} / r_{h i}=3.0$. Within the designs of minimum cruise drag for $L_{n a c} / r_{h i}=2.8$, $\Delta C_{D-M a c h}$ increases from 0.0076 to 0.0228 across the range of $r_{t e} / r_{h i}$ from 1.0 to 0.89 (Fig. 6b). A nacelle design with $\Delta C_{D-M a c h} \approx 0$ but a large $C_{D-\text { cruise }}$ of 0.0295 was found for the $L_{n a c} / r_{h i}=2.8$ and $r_{t e} / r_{h i}=1.0$ configuration. For the other trailing edge radii $\left(r_{t e} / r_{h i}=0.95\right.$ and 0.89$)$ the MOO did not identify any nacelle with $\Delta C_{D-M a c h} \approx 0$. The lowest achievable $\Delta C_{D-M a c h}$ with a $r_{t e} / r_{h i}=0.95$ was 0.0012 and a concomitant $C_{D-c r u i s e}=0.0329$. On the other hand, the $r_{t e} / r_{h i}=0.89$ configuration has a minimum $\Delta C_{D-M a c h}=0.02462$ with an associated $C_{D-\text { cruise }}=0.0321$.

The effects of the nacelle compactness are further highlighted for the more aggressive configurations of $L_{n a c} / r_{h i}=2.6$ and 2.4 (Fig. 5). For a fixed $L_{n a c} / r_{h i}$ $=2.6$ the MOO showed that the minimum drag increases by $35 \%: C_{D-\text { cruise }}$ $=0.0257,0.0272$ and 0.0347 for $r_{t e} / r_{h i}=1.0,0.95$ and 0.89 , respectively (Fig. 6a). The most aggressive configuration in terms on fan cowl length considered in 
this investigation, $L_{n a c} / r_{h i}=2.4$, confirms the inherent challenges of designing short nacelles. The cruise drag increased by $24 \%$ in the range of $r_{t e} / r_{h i}$ from 1.0 to $0.95, C_{D-\text { cruise }}=0.0253$ and 0.0313, with a $\Delta C_{D-\text { Mach }}=0.0150,0.0310$ (Fig. 6a and 6b). During the optimisation of the most compact case $\left(L_{n a c} / r_{h i}\right.$ $=2.4$ and $\left.r_{t e} / r_{h i}=0.89\right)$, all designs exhibited a peak isentropic Mach number above 1.3. This is caused by the overall amount of the fan cowl curvature of this configuration, which induces a strong acceleration over the lip that terminates in a strong shock wave and large wave drag.

This set of MOO reveals the trade-off in minimum achievable nacelle drag for the pertinent design variables of $L_{n a c} / r_{h i}$ and $r_{t e} / r_{h i}$. Across all the design space there is a $37 \%$ variation in terms of $C_{D \text {-cruise }}$ (Fig. 6a). For a fixed fan cowl length $\left(L_{n a c} / r_{h i}\right)$, the reduction of cruise drag can be obtained by consistently moving outboards the trailing edge location towards $r_{t e} / r_{h i}=1.0$. On the other hand, there is an optimum fan cowl length at a fixed trailing edge radius. For example, for a compact nacelle of $r_{t e} / r_{h i}=0.89$, the minimum cruise drag was found for a $L_{n a c} / r_{h i}$ of about 2.8 , which reduced to $L_{n a c} / r_{h i}=2.6$ for the more outboard trailing edge location of $r_{t e} / r_{h i}=1.0$. This is caused by the trade-off between wetted area and fan cowl curvature: while short nacelles have low wetted area, they present large wave drag caused by the increment of the fan cowl curvature.

\subsubsection{Impact of the design variables on the nacelle performance}

The result of a multi-objective optimisation is a set of non-dominated solutions. Therefore, it is difficult to select a specific individual from a Pareto front for problems with several objective functions. Parallel coordinate plots represent the regions of the design space where the non-dominated solutions are concentrated. Thus, this type of representation can be useful for two main purposes. Firstly, to check if the non-dominated solutions are not reaching the 


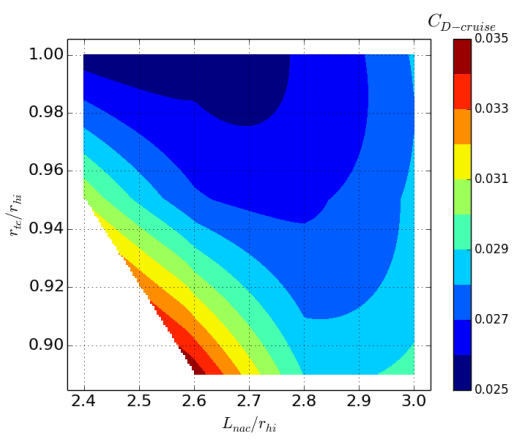

(a) $C_{D \text {-cruise }}$ across the design space

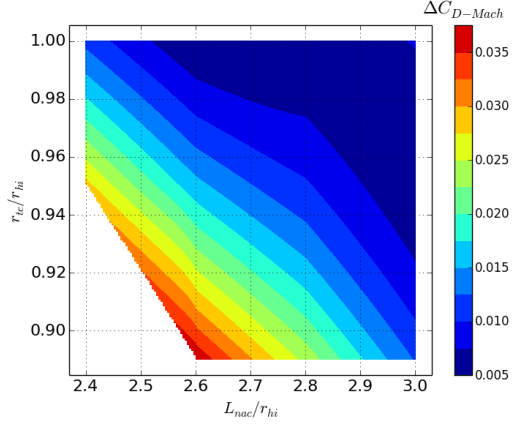

(b) $\Delta C_{D-M a c h}$ across the design space

Figure 6: $C_{D-\text { cruise }}$ and $\Delta C_{D-M a c h}$ changes across the DOE for the fan cowl with the lowest $C_{D-\text { cruise }}$ identified throughout the optimisation routine

bounds of the design variables, which assures a proper bounding of the problem. Secondly, to identify the parts of the design space where the non-dominated solutions are located. Figure 7 presents the parallel coordinates plot, colored by $C_{D-\text { cruise }}$, across the design space investigated. Each design variable has been normalized, where the value 0 refers to its lower bound and 1 to the upper bound. Relative to a conventional design with $L_{n a c} / r_{h i}=3.0$ and $r_{t e} / r_{h i}=$ 1.0, the scatter of the design variables for the individuals in the Pareto front reduces as the trailing edge moves inboards towards $r_{t e} / r_{h i}=0.89$, which highlights the difficulties to design feasible compact nacelles (Fig. 7). The same tendency is encountered when the fan cowl length is shortened to $L_{n a c} / r_{h i}=$ 2.4 at fixed trailing edge radius of $r_{t e} / r_{h i}=1.0$. Across all the design space investigated, the parallel coordinates plot of the nacelle design variables $f_{\max }$, $r_{\max } / r_{h i}$ and $f_{i f}$ are in a narrower band than the variables $y_{T E}^{\prime \prime}$ and $\beta_{n a c}$. It highlights the first order impact of this three design variables $\left(f_{\max }, r_{\max } / r_{h i}\right.$ and $\left.f_{i f}\right)$ in the performance metrics considered in this work. This can be of interest in the design of viable compact nacelles where there are also additional design considerations to be taken into account. For example, there is the need to accommodate auxiliary systems within the nacelle as well as the provision of 
sufficient ground clearance and installation on the aircraft. For these compact nacelles, which may be viable aerodynamically, the substantially constrained design space for key parameters $\left(f_{\max }, r_{\max } / r_{h i}\right.$ and $\left.f_{i f}\right)$ will affect the overall nacelle design and performance. For the dominant designs, optimum individuals in terms of cruise drag are obtained with relatively low values $f_{\max }, r_{\max } / r_{h i}$ and $f_{i f}$. The nacelles designs with high values of these three design variable $\left(f_{\max }, r_{\max } / r_{h i}\right.$ and $\left.f_{i f}\right)$ present large penalties in cruise drag (Fig. 7) but are optimum in terms of the other performance metrics considered $\left(\Delta C_{D-M a c h}\right.$ or $\left.C_{D-\text { spill }}\right)$.
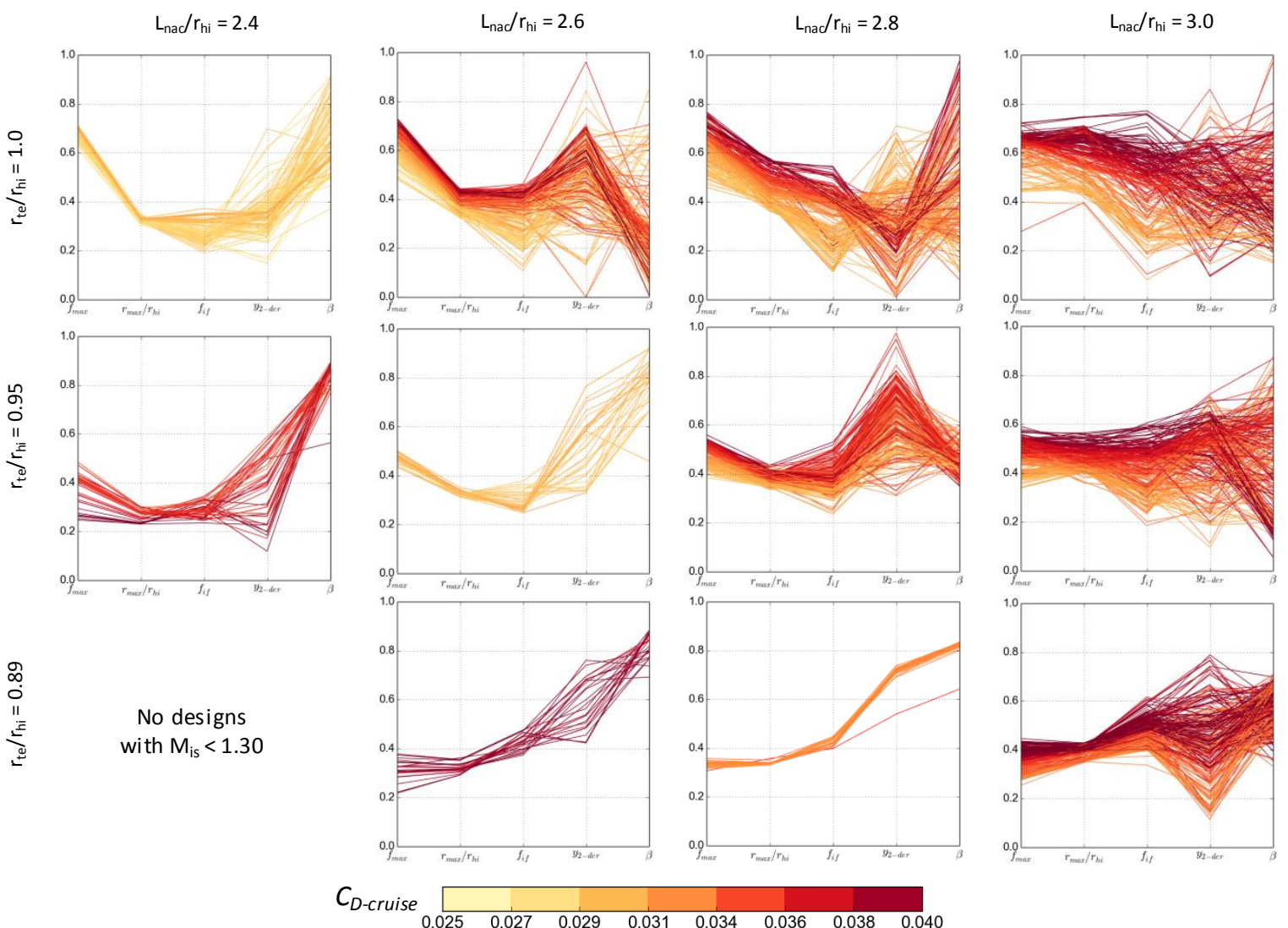

Figure 7: Parallel coordinates plot, collored by $C_{D-c r u i s e}$, across the representative design space of VHBR engines 
The trends in the design space for nacelles with the minimum $C_{D-\text { cruise }}$ for different fan cowl lengths and trailing edge location was assessed in terms of the key parameters $r_{\max } / r_{h i}, f_{\max }, f_{i f}$ and $\beta_{n a c}$ (Fig. 8). This is a useful representation to quantify the changes of the intuitive nacelle design variables employed in the proposed parametrisation with iCSTs. It allows deriving basic nacelle design guidelines at a preliminary stage of the design process. The design variable $r_{\max } / r_{h i}$ reduces with the nacelle length and trailing edge radius (Fig. 8a), which decreases the fan cowl curvature and, therefore, the acceleration over the fan cowl and its associated wave drag. There is only a $3 \%$ variation of $r_{\max } / r_{h i}$ for the nacelle designs with minimum cruise drag across the design space. In contrast to $r_{\max } / r_{h i}$, the parameter $f_{\max }$ presents a variability of $25.7 \%$ in the design space (Fig. 8b). This design variable increases when the fan cowl length is shortened or the trailing edge radius moves outboards towards $r_{t e} / r_{h i}=1.0$. The non-dimensional radius of curvature at the highlight $\left(f_{i f}\right)$ is constant at fixed $r_{t e} / r_{h i}$ (Fig. 8c). This is caused by its normalised definition in which at constant $r_{t e} / r_{h i}$, the changes in the optimum design variables $f_{\max }$, $r_{\max } / r_{h i}$ and $r_{i f}$ lead to a minimal variation of $f_{i f}$. Lastly, the boat-tail presents the largest scatter of all design variables across the design space, with a variation of $40 \%$ (Fig. 8 d), which confirms the mostly second order impact of this design variable in $C_{D-\text { cruise }}$.

\section{Conclusions}

A method for the multi-objective optimisation of nacelle aerolines for future aero-engines has been developed. The present framework comprises nacelle geometry definitions using intuitive Class Shape Transformations, automatic grid generation, a Favre Average Navier Stokes flow solver, automatic post-processing of the nacelle key metrics and a genetic algorithm. The methodology proposed in 


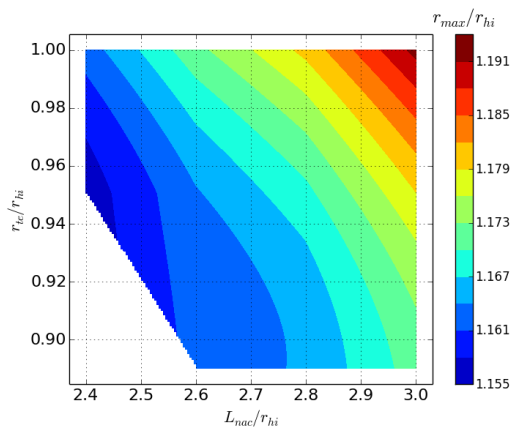

(a) $r_{\max } / r_{h i}$ across the design space

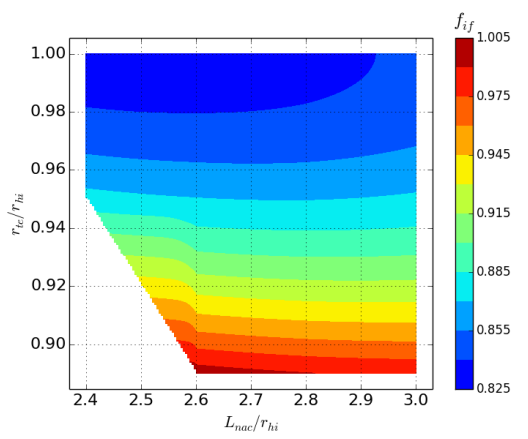

(c) $f_{\text {if }}$ across the design space

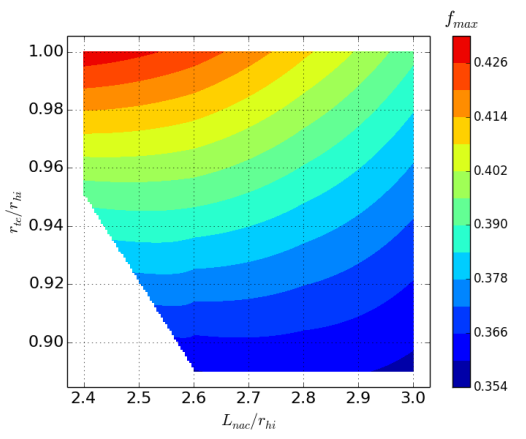

(b) $f_{\max }$ across the design space

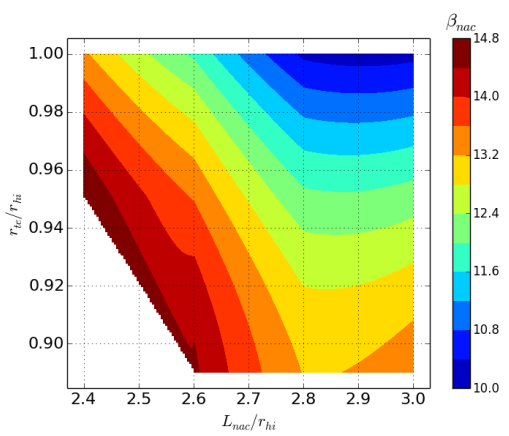

(d) $\beta_{n a c}$ across the design space

Figure 8: Design variable changes across the DOE for the fan cowl with the lowest $C_{D-\text { cruise }}$ identified throughout the optimisation routine 
this paper has been successfully validated with the available experimental data. It has been shown that the framework can be employed to investigate nacelle aero-lines within the new design space for the future aero-engines. Furthermore, it has been demonstrated the challenges of designing feasible compact nacelles and the changes in the design space for different nacelles length and trailing edge radii have been quantified. It has been found that the Pareto front representation combined with the Parallel plot coordinates can identify the feasible regions of the design space and quantify the dominant design parameters. The combination of both representations has been employed to limit the viable design space and to highlight nacelle design sensitivities for the future aero-engines at an early stage of the design process. Hence, the proposed framework constitutes an useful tool for the design of optimum compact nacelles. 


\section{References}

[1] N. T. Birch, 2020 Vision: The Prospects for Large Civil Aircraft Propulsion, The Aeronautical Journal 104 (2000) 347-352.

[2] N. Cumpsty, Preparing for the Future: Reducing Gas Turbine Environmental Impact - IGTI Scholar Lecture, Journal of Turbomachinery 132 (2010) 041017-1 - 041017-17.

[3] M. Waters, E. Schairer, Analysis of Turbofan Propulsion System Weight and Dimensions, Tech. Rep. TM X-73, NASA (1977).

[4] J. Marx, Installation Features of Advances, Very High Bypass Turbofan Propulsion Systems, in: AIAA/AHS/ASEE Aircraft Design, Systems and Operations Conference, no. AIAA Paper 2015-3778, Orlando, Florida, USA, 1989.

[5] D. Dagget, Ultra-Efficient Engine Technology Systems Integration and Environmental Assessment, Tech. Rep. CR-2002-211754, NASA (2002).

[6] D. Daggett, S. Brown, R. Kawai, Ultra-Efficient Engine Diameter Study, Tech. Rep. CR-2003-212309, NASA (2003).

[7] J. Seddon, E. Goldsmith, Intake Aerodynamics, IAA Education Series, Vol. 2, American Institute of Aeronautics and Astronautics, 1999.

[8] A. Peters, Z. S. Spakovszky, W. K. Lord, B. Rose, Ultrashort Nacelles for Low Fan Pressure Ratio Propulsors, Journal of Turbomachinery 137 (2) (2015) 021001-1 - 021001-14.

[9] K. Vunnam, R. Hoover, Modeling of Inlet Distortion using a Combined Turbofan and Nacelle Inlet Model during Crosswind and Low Speed Forward Operation, in: Proceedings of ASME Turbo Expo 2011, GT3011-46466, Rome, Italy, 2011. 
[10] M. Robinson, D. MacManus, A. Heidebrecht, An Optimization Method for Nacelle Design, in: AIAA SciTech Forum, 55th AIAA Aerospace Sciences Meeting, no. AIAA Paper 2015-3778, Orlando, Florida, USA, 2017.

[11] D. Mitchell, Estimation of Spillage Drag for a Wide Range of Axisymmetric Intakes at $\mathrm{M}<1$, Tech. Rep. ESDU-84004, ESDU (1984).

[12] A. M. Carr, Estimation of Windmilling Drag and Airflow of Tubo-Jet and Turbo-Fan Engines, Tech. Rep. ESDU-81009, ESDU (November 1981).

[13] M. Martin, E. Andres, C. Lozano, E. Valero, Volumetric B-Splines Shape Parametrization for Aerodynamic Shape Design, Aerospace Science and Technology 37 (2014) 26-36.

[14] Y. Zhang, X. Fang, H. Chen, S. Fu, Z. Duan, Y. Zhang, Supercritical Natural Laminar Flow Airfoil Optimization for Regional Aircraft Wing Design, Aerospace Science and Technology 43 (2015) 152-164.

[15] P. Vecchia, E. Daniele, E. D'Amato, An Airfoil Shape Optimization Technique Coupling PARSEC Parameterization and Evolutionary Algorithm, Aerospace Science and Technology 32 (2014) 103-110.

[16] D. A. Masters, N. J. Taylor, T. C. S. Rendall, C. B. Allen, D. J. Poole, Geometric Comparison of Aerofoil Shape Parameterization Methods, AIAA Journal 55, (5) (2017) 1575-1589.

[17] T. Baklacioglu, Modeling the Fuel Flow-Rate of Transport Aircraft During Flight Phases Using Genetic Algorithm-Optimized Neural Networks, Aerospace Science and Technology 49 (2016) 52-62.

[18] M. Albert, D. Bestle, Aerodynamic Design Optimization of Nacelle and Intake, in: Proceedings of ASME Turbo Expo 2013: Turbine Technical Conference and Exposition, Virginia Beach, VA, USA, 2013. 
[19] H. Toubin, I. S. E. Dim, M. Meheut, Multipoint Aerodynamic High Fidelity Shape Optimization of an Isolated Engine Nacelle, in: AIAA SciTech Forum 52nd Aerospace Sciences Meeting, Virginia Beach, VA, USA, 2014.

[20] X. Fang, Y. Zhang, H. Chen, Transonic Nacelle Aerodynamic Optimization Based on Hybrid Genetic Algorithm, in: 17th AIAA/ISSMO Multidisciplinary Analysis and Optimization Conference, no. AIAA 2016-3833, Virginia Beach, VA, USA, 2016.

[21] S. Li, Y. Zhong, A Turbofan-Engine Nacelle Shape Design and Optimization Method for Natural Laminar Flow Control, in: Proceedings of ASME Turbo Expo 2016: Turbomachinery Technical Conference and Exposition, GT2016-57463, no. AIAA Paper No. 2015-3779, Orlando, Florida, USA, 2016.

[22] H. Bayraktar, F. Turalioglu, A Kriging-based Approach for Locating a Sampling Site in the Assessment of Air Quality, Stochastic Environmental Research and Risk Assessment 19 (2005) 301-305.

[23] J. H. S. Finchman, M. I. Friswell, Aerodynamic Optimisation of a Camber Morphing Aerofoil, Aerospace Science and Technology 43 (2015) 245-255.

[24] I. Goulos, T. Stankowski, D. MacManus, P. Woodrow, C. Sheaf, Civil Turbofan Engine Exhaust Aerodynamics: Impact of Bypass Nozzle After-Body Design, Aerospace Science and Technology 71 (2018) 85-95.

[25] Q. Wang, Q. Zhao, Rotor Airfoil Profile Optimization for Alleviating Dynamic Stall Characteristics, Aerospace Science and Technology 72 (2018) $502-515$.

[26] T. S. Crum, D. E. Yates, T. L. Andrew, N. O. Stockman, Low Speed Test 
Results of Subsonic, Turbofan Scarf Inlets, in: AIAA/SAE/ASME/ASEE 29th Joint Propulsion Conference and Exhibit, AIAA 93-2301, 1993.

[27] B. M. Kulfan, Recent Extensions and Applications of the 'CST' Universal Parametric Geometry Representation Method, The Aeronautical Journal 114, (1153) (2010) 157-176.

[28] Ansys Inc., 275 Technology Drive, Canonsburg, PA 15317, ANSYS ICEM CFD Tutorial Manual.

[29] Ansys Inc., 275 Technology Drive, Canonsburg, PA 15317, ANSYS FLUENT User's Guide.

[30] K. Deb, A. Pratap, S. Agarwal, T. Meyarivan, A Fast and Elitist Multiobjective Genetic Algorithm: NSGA-II, IEEE Transactions on Evolutionary Computation 6, (2) (2002) $182-197$.

[31] V. Sripawadkul, M. Padulo, M. Guenov, A Comparison of Airfoil Shape Parameterization Techniques for Early Design Optimization, in: 13th AIAA/ISSMO Multidisciplinary Analysis Optimization Conference, no. AIAA 2010-9050, Fort Worth, Texas, 2010.

[32] T. Zhang, Z. Wang, W. Huang, L. Yan, Parameterization and Optimization of Hypersonic-Gliding Vehicle Configurations During Conceptual Design, Aerospace Science and Technology 58 (2016) 225-234.

[33] C. Liu, Y. Duan, J. Cai, J. Wang, Application of the 3D Multi-Block CST Method to Hypersonic Aircraft Optimization, Aerospace Science and Technology 50 (2016) 295-303.

[34] R. Christie, A. Heidebrecht, D. G. MacManus, An Automated Approach to Nacelle Parameterisation Using Intuitive Class Shape Transformation 
Curves, Journal of Engineering for Gas Turbines and Power 139 (2017) 062601-1-062601-9.

[35] I. Goulos, T. Stankowski, J. Otter, D. MacManus, N. Grech, C. Sheaf, Aerodynamic Design of Separate-Jet Exhausts for Future Civil Aero-Engine, Part 1: Parametric Geometry Definition and CFD Approach, ASME J. Eng. Gas Turbines and Power 138, (8) (2016) 081201.

[36] P. Roache, A Method for Uniform Reporting of Grid Refinement Studies, Journal of Fluids Engineering 116 (3) (1994) 405-413.

[37] M. Langley, The Design of Axisymmetric Cowls for Podded Nacelles for High Bypass Ratio Turbofan Engines, Tech. Rep. No. 3846, Aeronautical Research Council (1979).

[38] Study Group MIDA, Guide to in-flight thrust measurement of turbojets and fan engines, Tech. Rep. AGARD-AG-237, Advisory Group for Aerospace Research and Development (1979).

[39] R. Christie, S. Ramirez, D. G. MacManus, Aero-engine installation modelling and the impact on overall flight performance, in: In Advanced Aero Concepts, Design and Operations Conference, 2014.

[40] T. Stankowski, D. G. MacManus, C. Sheaf, R. Christie, Aerodynamics of Aero-Engine Installation, Proceedings of the Institution of Mechanical Engineers, Part G: Journal of Aerospace Engineering 230 (14) (2016) 26732692. 
2019-02-19

\section{Multi-objective optimisation of short nacelles for high bypass ratio engines}

Tejero, Fernando

Elsevier

Tejero F, Robinson M, MacManus DG, Sheaf C. (2019) Multi-objective optimisation of short nacelles for high bypass ratio engines. Aerospace Science and Technology, Volume 91, August 2019, pp. 410-421

https://doi.org/10.1016/j.ast.2019.02.014

Downloaded from Cranfield Library Services E-Repository 